\title{
Vorsicht bei Vorschlägen zur Interpretation von Regressionen mit Interaktionsvariablen
}

\author{
Reinhard Schunck $\cdot$ Natascha Nisic
}

Online publiziert: 7. Mai 2020

(C) Der/die Autor(en) 2020

Zusammenfassung Die zentrale Idee einer Interaktion besteht darin, einen konditionalen Effekt zu modellieren, d. h. der Effekt einer Variablen $\left(x_{i}\right)$ auf die abhängige Variable $\left(y_{i}\right)$ hängt vom Wert einer anderen Variablen $\left(z_{i}\right)$ ab. Mayerl und Urban diskutieren in ihrer Abhandlung die Eigenschaften von Haupt- und Interaktionseffekten und welche Fallstricke sich bei deren Interpretation ergeben können. Leider vermitteln Mayerl und Urban den Eindruck, als ob Haupt- und Interaktionseffekte sinnvoll isoliert und damit unabhängig voneinander interpretiert werden können. Auch wenn dies in Ausnahmefällen zutreffen kann, ist dies allgemein nicht sinnvoll. Wir zeigen, dass eine isolierte oder getrennte Interpretation von Haupt- und Interaktionseffekten irreführend sein kann, auch wenn die von Mayerl und Urban diskutierten Probleme nicht vorliegen. Weil es sich bei Koeffizienten in Regressionsmodellen mit Interaktionstermen um konditionale Effekte handelt, ist eine Darstellung und Interpretation ratsam, die alle an der Interaktion beteiligten Kovariaten gleichzeitig einbezieht. Empfohlen wird eine (grafische) Darstellung, die Auskunft darüber gibt, wie der Effekt von $x_{i}$ auf $y_{i}$ über den Wertebereich von $z_{i}$ variiert.

Schlüsselwörter Lineare Regression · Logistische Regression · Konditionale Effekte $\cdot$ Interaktionseffekte $\cdot$ Haupteffekte $\cdot$ Marginale Effekte

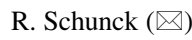

Fakultät für Human- und Sozialwissenschaften, Bergische Universität Wuppertal

Gaußstr. 20, 42119 Wuppertal, Deutschland

E-Mail: schunck@uni-wuppertal.de

\section{N. Nisic}

Institut für Ernährung, Konsum, Gesundheit, Universität Paderbon

Warburger Str. 100, 33098 Paderborn, Deutschland

E-Mail: nnisic@mail.upb.de 


\title{
Caution with of Suggestions for Interpreting Regressions with Interactions
}

\begin{abstract}
The central idea of an interaction is to model a conditional effect, i.e., the effect of one variable $\left(x_{i}\right)$ on the dependent variable $\left(y_{i}\right)$ depends on the values of another variable $\left(z_{i}\right)$. In their article, Mayerl and Urban discuss the properties of main and interaction effects and the pitfalls that can arise in their interpretation. Unfortunately, Mayerl and Urban give the impression that main and interaction effects can be meaningfully interpreted independently of one another. Although this may be true in exceptional cases, it is not generally recommendable. We show that an isolated or independent interpretation of main and interaction effects can be misleading, even if the specific problems discussed by Mayerl and Urban are not present. Because the effects of covariates that are part of the interaction are conditional effects, it is advisable to present and interpret them in a way that includes all covariates involved in the interaction. We recommend a (graphical) representation that gives information about how the effect of $x_{i}$ on $y_{i}$ varies over the range of $z_{i}$.
\end{abstract}

Keywords Linear Regression · Logistic Regression · Conditional Effects · Interaction Effects · Main Effects · Marginal Effects

\section{Einleitung}

In der Ausgabe 02/2019 der Kölner Zeitschrift für Soziologie und Sozialpsychologie haben Mayerl und Urban (2019) eine ausführliche Abhandlung zum Verhalten von Haupt- und Interaktionseffekten in linearen Regressionsmodellen publiziert. Auch wenn die Autoren mehr Sorgfalt bei der Auswahl der Negativbespiele hätten walten lassen können, zumindest bei Nisic (2010) und Schunck (2016) tauchen die von Mayerl und Urban (2019) beschriebenen Probleme zwar auf, die Interaktionen werden aber nicht fehlerhaft interpretiert ${ }^{1}$, wäre dies kein Grund für einen Kommentar.

Zentraler Gegenstand der Abhandlung von Mayerl und Urban (2019, S. 135) sind „methodische Fallstricke“ bei der Interpretation von Haupteffekten aus Regressionsmodellen mit Interaktionstermen. Mayerl und Urban (2019) illustrieren sehr detailliert, wie die geschätzten Haupteffekte vom Wertebereich oder der Skalierung der zugrunde liegenden Variablen abhängen. Als besonders problematisch wird beschrieben, wenn der Wertebereich einer an der Interaktion beteiligten Variablen die 0 nicht umfasst. In dieser Situation bezieht sich der geschätzte Haupteffekt der an-

\footnotetext{
1 Im Artikel von Nisic (2010) wird der durch die Aufnahme des Interaktionsterms hervorgerufene Vorzeichenwechsel des Haupteffekts explizit als konditionaler Effekt interpretiert. Dieser bezieht sich zudem auf einen inhaltlich sinnvollen Wert, da die an der Interaktion beteiligte kontinuierliche Variable (Bildungsjahre) zuvor entsprechend zentriert wurde ( $0=$ Hauptschulabschluss) (s. Nisic 2010, S. 532, 537 u. 539 sowie Tab. 3, Modell 1 und 2). Zudem werden Haupt- und Interaktionseffekt bei der Interpretation gemeinsam betrachtet, indem auf den (vorhergesagten) positiven Effekt für Frauen mit Abitur verwiesen wird (vgl. S. 539) - wenn auch der Interaktionseffekt leider nicht grafisch dargestellt ist, wie in dieser Abhandlung im Weiteren vorgeschlagen; im Artikel von Schunck (2016) wird der gemeinsame Effekt grafisch dargestellt.
} 
deren an der Interaktion beteiligten Variablen auf eine Situation, die es nicht gibt (Mayerl und Urban 2019, S. 145, 146, 148). Soweit lässt sich den Ausführungen auch folgen. Leider vermittelt der Beitrag in unseren Augen den Eindruck, dass Haupt- oder Interaktionseffekte sinnvoll isoliert bzw. unabhängig voneinander interpretiert werden könnten (Mayerl und Urban 2019, siehe bspw. S. 136, 138, 147, $148,149-150,152)^{2}$. Dem möchten wir widersprechen. In Regressionen mit Interaktionen handelt es sich um konditionale Effekte. D.h. der Effekt einer Kovariate auf die abhängige Variable hängt vom Wert einer anderen Kovariaten ab. Entsprechend sollten Haupt- und Interaktionseffekte gemeinsam interpretiert werden (siehe bspw. Aiken und West 1991; Braumoeller 2004; Brambor et al. 2006; Clark et al. 2006; Berry et al. 2012). Mayerl und Urbans Vorschläge für die Forschungspraxis (Mayerl und Urban 2019, S. 151-152) sind unseres Erachtens daher nicht zielführend und lösen die grundsätzliche Herausforderung hinsichtlich der Interpretation von Interaktionen nicht.

Wie wenig sinnvoll eine isolierte oder getrennte Interpretation von Haupt- und Interaktionseffekten ist, soll im Folgenden dargelegt werden. Zunächst zeigen wir dies an der Herleitung der marginalen Effekte und illustrieren dies mit simulierten Daten. Anschließend machen wir knapp einige Vorschläge für die Forschungspraxis.

\section{Illustration}

Gehen wir zunächst von einem additiven linearen Modell ohne Interaktion mit zwei intervallskalierten Kovariaten aus:

$$
y_{i}=\beta_{1}+\beta_{2} x_{i}+\beta_{3} z_{i}+\varepsilon_{i}
$$

Die partielle Ableitung von (1) nach $x_{i}$ - die also den marginalen Effekt von $x_{\mathrm{i}}$ angibt - ist:

$$
\frac{\partial y_{i}}{\partial x_{i}}\left[\beta_{1}+\beta_{2} x_{i}+\beta_{3} z_{i}+\varepsilon_{i}\right]=\beta_{2}
$$

Im linearen additiven Modell ist der marginale Effekt gleich dem Steigungskoeffizienten $\left(\beta_{2}\right)$. Er ist konstant und seine Interpretation unproblematisch: Er gibt die erwartete Auswirkung einer Änderung der Kovariaten $x_{i}$ auf die abhängige Variable $y_{i}$ an und entspricht dem durchschnittlichen marginalen Effekt über alle Beobachtungen (dem sog. ,average marginal effect“ - AME) (Cameron und Trivedi 2005,

\footnotetext{
2 Dieser Eindruck entsteht insbesondere dadurch, dass Mayerl und Urban (2019) den Fokus fast ausschließlich auf die Interpretation der Haupteffekte legen und Haupt- und Interaktionseffekte isoliert diskutieren (S. 138, 140f.), während der konditionale Charakter von Interaktionseffekten und die sich daraus ableitende Notwendigkeit der gemeinsamen Interpretation von Haupt- und Interaktionseffekt kaum zur Sprache kommen (Mayerl und Urban 2019, S. 147, 153, 154) und bei den Empfehlungen für die Forschungspraxis gänzlich fehlen (Mayerl und Urban 2019, S. 151-152).
} 
S. 122). Nun nehmen wir an, dass der Effekt von $x_{i}$ auf $y_{i}$ vom Wert von $z_{i}$ abhängt. Entsprechend erweitern wir das Modell um eine Interaktion zwischen $x_{i}$ und $z_{i}$ :

$$
y_{i}=\beta_{1}+\beta_{2} x_{i}+\beta_{3} z_{i}+\beta_{4} x_{i} z_{i}+\varepsilon_{i}
$$

Wir konzentrieren uns im Folgenden auf den geschätzten Effekt von $x_{i}$ auf $y_{i}$ und behandeln $z_{i}$ als Moderatorvariable. Da multiplikative Interaktionsmodelle symmetrisch sind, muss eine solche Entscheidung theoretisch begründet werden. Im Gegensatz $\mathrm{zu}(1)$ ist der marginale Effekt von $x_{i}$ in einem linearen Modell mit Interaktion:

$$
\frac{\partial y_{i}}{\partial x_{i}}\left[\beta_{1}+\beta_{2} x_{i}+\beta_{3} z_{i}+\beta_{4} x_{i} z_{i}+\varepsilon_{i}\right]=\beta_{2}+\beta_{4} z_{i}
$$

Es ist offensichtlich, dass der marginale Effekt von $x_{i}$ von $z_{i}$ abhängt. Wie Mayerl und Urban (2019) richtig feststellen, bezieht sich der geschätzte Haupteffekt von $x_{\mathrm{i}}$ allein auf die Situation wenn $z_{i}=0$. Das von Mayerl und Urban (2019) ausführlich diskutierte Problem (s. hierzu auch schon Allison 1977; Aiken und West 1991) stellt sich nun, wenn der Wertebereich der Variable $z_{i}$ die 0 nicht umfasst. Dann bezieht sich der geschätzte Haupteffekt $\left(\widehat{\beta}_{2}\right)$ auf eine Situation, die es nicht gibt. Entsprechend ,unsinnig“ wäre der Schätzer $\left(\widehat{\beta}_{2}\right)$ oder dessen Interpretation.

Ist dies der Fall, schlagen Mayerl und Urban (Mayerl und Urban 2019, S. 152) vor, entweder zu reskalieren oder zu zentrieren: ,,[ob] bei der Schätzung von Interaktionsmodellen rekodierte Variablen mit gültigen 0-Wert eingesetzt werden sollen (es könnte z. B. die Skala [+1 bis +5$]$ in die Skala [0 bis +4$]$ oder die Skala [ -2 bis +2$]$ überführt werden) oder die mittelwertzentrierte Version verwendet, bleibt gänzlich den Anwendern überlassen. Allerdings hat die mittelwertzentrierte Lösung insofern den Vorteil, dass dabei der Variablenwert von 0 immer eine gut zu interpretierende ,durchschnittliche Variablenausprägung ' bezeichnet" (Mayerl und Urban 2019. S. 152).

Betrachten wir zunächst den ersten Vorschlag, eine Reskalierung von $z_{i}$, sodass der Wertebereich nun 0 umfasst. Kann der Haupteffekt von $x_{i}$ nun sinnvoll isoliert, d. h. ohne Bezugnahme auf $z_{i}$, interpretiert werden? Zwar bezieht sich der geschätzte Haupteffekt $\left(\widehat{\beta}_{2}\right)$ nun nicht mehr auf eine Situation, die es nicht gibt. Trotzdem ist eine isolierte Interpretation des Haupteffekts keinesfalls zu empfehlen - unabhängig davon, ob $z_{i}$ die 0 umfasst, weil die ursprüngliche Variable bereits einen sinnvollen oder natürlichen Nullpunkt hat oder weil reskaliert wurde. Dies liegt eben daran, dass es sich bei einer Interaktion um einen konditionalen Effekt handelt. Der Effekt von $x_{i}$ auf $y_{i}$ kann über den Wertebereich von $z_{i}$ variieren, wie der marginale Effekt deutlich zeigt (4). Zwar ist der Effekt von $x_{i}$ auf $y_{i}$ prinzipiell interpretierbar - er bezieht sich aber nur auf eine Situation $\left(z_{i}=0\right)$ und ist damit wenig informativ, falls wir einen konditionalen Effekt annehmen.

Es bleibt die Frage, ob der zweite Vorschlag, die Mittelwertzentrierung - die von Mayerl und Urban bevorzugte Methode (Mayerl und Urban 2019, S. 152) Abhilfe verschafft. Wie bei Mayerl und Urban (2019) veranschaulicht, führt die Mittelwertzentrierung der Kovariaten in (3) dazu, dass der geschätzte Haupteffekt $\left(\widehat{\beta}_{2}\right)$ den mittleren Effekt von $x_{i}$ auf $y_{i}$ über den gesamten Wertebereich von $z_{i}$ 
angibt (Brambor et al. 2006, S. 71-73; Balli und Sørensen 2013, S. 583; Cohen et al. 2003, S. $261 \mathrm{ff}$.). Entscheidend für die Interpretationsmöglichkeit des Haupteffekts als AME ist allerdings - hier sind Mayerl und Urban (2019) in ihrer Darstellung nicht präzise -, dass die andere am Interaktionseffekt beteiligte Variable zentriert ist (d.h. der Moderator $z_{i}$ ). Der interpretierte Haupteffekt selbst muss dazu nicht auf einer zentrierten Variablen beruhen. Hintergrund für diese Interpretationsvariante ist der Umstand, dass im linearen Modell der durchschnittliche Marginaleffekt (AME) dem Marginaleffekt am Mittelwert der Daten (,marginal effect at mean“ - MEM) entspricht (Cameron und Trivedi 2005, S. 122). Ist $z_{i}$ zentriert, gibt der Haupteffekt von $x_{i}$ an der Stelle 0 somit den Effekt von $x_{i}$ für eine durchschnittliche Variablenausprägung von $z_{i}$ wieder (MEM) oder eben den durchschnittlichen Effekt von $x_{i}$ auf $y_{i}$ über $z_{i}$ (AME). Dieser Effekt ist aber mitnichten ,immer gut zu interpretieren“ (Mayerl und Urban 2019. S. 152). Denn aus der Äquivalenz von AME und MEM im linearen Modell folgt auch, dass die Haupteffekte im zentrierten Modell den marginalen Effekten (AMEs) aus dem additiven Modell ohne Interaktion entsprechen (Balli und Sørensen 2013, S. 583). ${ }^{3}$ Die Interpretation des Haupteffekts im zentrierten Modell enthält somit keinen Informationsgewinn gegenüber dem additiven Modell.

Die isolierte Interpretation eines Haupteffekts ohne die Berücksichtigung des anderen Haupteffekts und des Interaktionseffekts kann zudem sehr irreführend sein, auch wenn der Wertebereich der Kovariaten die 0 umfasst oder die Kovariaten zentriert wurden. Um dies zu illustrieren, haben wir auf Basis der Modelle (1) und (3) einen Datensatz mit 1000 Beobachtungen simuliert. ${ }^{4}$ Wir haben folgende Werte gesetzt: $x_{i} \sim N(1,1), z_{i}$ ist gleichverteilt im Intervall $[0,4]$ und $\varepsilon_{i} \sim N(0,1)$. $\beta_{1}=1, \beta_{2}=-2, \beta_{3}=1$ und $\beta_{4}=1$.

Der Zusammenhang ist grafisch in Abb. 1 dargestellt. Es ist leicht ersichtlich, dass der Effekt von $x_{i}$ auf $y_{i}$ über den Wertebereich von $z_{i}$ variiert: von einem negativen Effekt, wenn $z_{i}<1$, bis zu einem deutlich positiven, wenn $z_{i} \geq 3$. Wir haben es also mit einem konditionalen Effekt zu tun. In Tab. 1 sind die zugehörigen Schätzungen einer Regression angegeben, ohne Interaktion (Modell 1), mit Interaktion, unzentriert (Modell 2), mit Interaktion, nur $z_{i}$ zentriert (Modell 3) und mit Interaktion, beide Kovariaten zentriert (Modell 4).

Im Modell mit den unzentrierten Variablen (Modell 2) geben die Schätzer für die Haupteffekte von $\beta_{2}$ und $\beta_{3}$ den jeweiligen Effekt der Kovariate auf $y_{i}$ an, wenn die jeweils andere Kovariate den Wert 0 annimmt. Ist $z_{i}=0$, so ist der geschätzte Haupteffekt $\widehat{\beta}_{2}=-1,90$ (Modell 2). Wie sieht es nun mit der von Mayerl und Urban (2019, S. 152) bevorzugten Variante aus, der Mittelwertzentrierung? Auf Basis der zentrierten Variablen ist der geschätzte Haupteffekt von $\widehat{\beta}_{2}=0,01$. Im Mittel gibt es also keinen Effekt (Modell 4). An dieser Stelle wird ersichtlich, dass bei einer Mittelwertzentrierung die Haupteffekte (Modell 4) nun sehr nah an den Effekten der-

\footnotetext{
${ }^{3}$ Empirisch entsprechen die zentrierten Haupteffekte nicht immer exakt den Effekten aus dem additiven Modell.

4 Stata-Syntax ist über das Open Science Framework (https://osf.io/gky8x/) verfügbar. Andere Probleme, wie bspw. Heteroskedastizität, werden im Folgenden nicht behandelt. Die Abbildungen wurden mit den Grafik-Schemata „blindschemes“ (Bischof 2017) erstellt.
} 


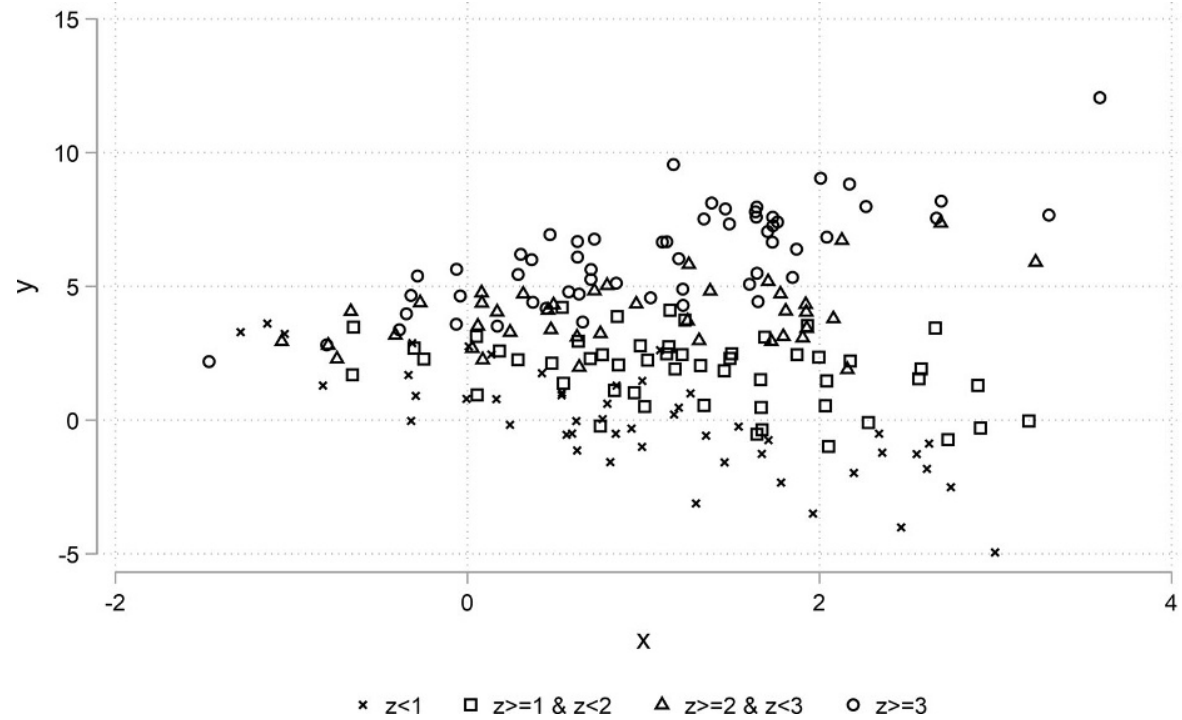

Abb. 1 Scatterplot von x und y. Hinweis: Es sind nur 200 der 1000 Datenpunkte geplottet, um die Übersichtlichkeit zu erhöhen

selben Kovariaten aus dem additiven Modell ohne Interaktion sind (Modell 1). Der Vergleich von Modellen (1), (3) und (4) verdeutlicht zudem, dass nur die Zentrierung des Moderators $\left(z_{i}\right)$ notwendig ist, um den mittleren Haupteffekt zu erhalten: Obwohl in Modell (3) nur $z_{i}$ zentriert ist, erhält man den mittleren Haupteffekt von $x_{i}$ auf $y_{i}\left(\widehat{\beta}_{2}=0,01\right)$. Eine isolierte Interpretation der Haupteffekte $\left(\widehat{\beta}_{2}\right.$ aus Modell 2 oder Modell 4) wäre in beiden Fällen formal korrekt. Im Vergleich mit Abb. 1 zeigt sich aber, wie wenig informativ bzw. irreführend die isolierte Betrachtung der Haupteffekte ist. Sie unterschlägt den konditionalen Zusammenhang zwischen $x_{\mathrm{i}}$ und $y_{i}$ in Abhängigkeit von $z_{i}$ - unabhängig davon, ob $z_{i}$ zentriert wurde oder der Haupteffekt für $z_{i}=0$ geschätzt wird.

Interessanterweise beschreiben Mayerl und Urban (2019, S. 152-153) diesen Sachverhalt selbst. Sie ziehen unseres Erachtens aber eine falsche Schlussfolgerung, nämlich gänzlich auf die Interpretation der Haupteffekte zu verzichten: „Eine Konsequenz daraus, dass es sich bei den Haupteffekten in Interaktionsmodellen um konditionale Effekte handelt, kann auch sein, dass Haupteffekte in Interaktionsmodellen überhaupt nicht mehr interpretiert werden sollten“ (Mayerl und Urban 2019, S. 152-153). Oder ,[w]enn in einer Regressionsanalyse allein die Interaktion zwischen Effektvariablen inhaltlich interessiert und wenn die Haupteffekte substanziell ohne Bedeutung sind, können die Haupteffekte bei der Interpretation der Schätzergebnisse auch schlicht ignoriert werden." Stattdessen könne nur der Interaktionseffekt interpretiert werden, da dieser von der Skalierung der zugrunde liegenden Variablen unberührt bleibt (Mayerl und Urban 2019, S. 152). Es mag Situationen geben, in denen, theoretisch begründet, lediglich der Interaktionseffekt von Interesse sein kann und eine Interpretation vergleichsweise unkompliziert ist (wenn bspw. zwei dichotome Kovariaten interagiert werden). Meist ist aber eine gemein- 
Tab. 1 Regression (OLS)

\begin{tabular}{lllll}
\hline & $(1)$ & $(2)$ & $(3)$ & $(4)$ \\
& $\begin{array}{l}\text { Ohne Int } \\
\mathrm{b} / \mathrm{se}\end{array}$ & $\begin{array}{l}\text { Unzentriert } \\
\mathrm{b} / \mathrm{se}\end{array}$ & $\begin{array}{l}\mathrm{z} \text { zentriert } \\
\mathrm{b} / \mathrm{se}\end{array}$ & $\begin{array}{l}\mathrm{x} \text { und z zentriert } \\
\mathrm{b} / \mathrm{se}\end{array}$ \\
\hline $\mathrm{X}$ & $-0,02$ & $-1,90$ & $-0,01$ & $-0,01$ \\
& $(0,05)$ & $(0,06)$ & $(0,03)$ & $(0,03)$ \\
$\mathrm{z}$ & 1,96 & 1,02 & 1,02 & 1,96 \\
& $(0,04)$ & $(0,04)$ & $(0,04)$ & $(0,03)$ \\
$\mathrm{X} * \mathrm{z}$ & - & 0,98 & 0,98 & 0,98 \\
& - & $(0,03)$ & $(0,03)$ & $(0,03)$ \\
Konstante & $-0,90$ & 0,91 & 2,88 & 2,87 \\
& $(0,10)$ & $(0,08)$ & $(0,04)$ & $(0,03)$ \\
$N$ & 1000 & 1000 & 1000 & 1000 \\
\hline
\end{tabular}

Standardfehler in Klammern

same Betrachtung von Haupt- und Interaktionseffekten notwendig, um konditionale Zusammenhänge verstehen und die theoretischen Vorhersagen prüfen zu können.

Es bleibt festzuhalten, dass eine isolierte oder getrennte Interpretation der Hauptund Interaktionseffekte nicht sinnvoll ist oder, wie Brambor et al. (2006) feststellen, ,it makes little sense to talk about the unconditional or average effect of $\mathrm{X}$ on $\mathrm{Y}$ when you have a conditional hypothesis." Eine gemeinsame Betrachtung von Haupt- und Interaktionseffekten empfiehlt sich daher immer.

\section{Vorschläge für die Forschungspraxis}

Vorzuziehen ist eine Darstellung und Interpretation durch vorhergesagte Werte oder marginale Effekte, die alle an der Interaktion beteiligten Kovariaten einbezieht. Insbesondere ist dabei eine grafische Darstellung inhaltlich sinnvoller Wertebereiche von $x_{i}$ und $z_{i} \mathrm{zu}$ empfehlen, um die Interpretation $\mathrm{zu}$ erleichtern (Brambor et al. 2006; Bauer 2010; Mitchell 2008; zur allgemeinen Diskussion über die Darstellung von Ergebnissen aus statistischen Analysen siehe auch King et al. 2000). Dies ist in Abb. 2 veranschaulicht. Hier wurden exemplarisch drei Werte für $z_{i}$ gewählt, der Mittelwert sowie der Mittelwert plus/minus eine Standardabweichung; im Hintergrund wurde zusätzlich noch mithilfe eines Histogramms die empirische Verteilung von $x_{i}$ abgebildet. Die grafische Darstellung, die auf den gemeinsamen Effekten von $x_{i}$ und $z_{i}$ sowie deren Interaktion beruht, veranschaulicht den konditionalen Effekt von $x_{i}$ auf $y_{i}$. Bei einem geringen Wert von $z_{i}$ (Mittelwert minus eine Standardabweichung), zeigt sich ein negativer Zusammenhang zwischen $x_{i}$ auf $y_{i}$, beim mittleren Wert von $z_{i}$ zeigt sich kein Zusammenhang und bei einem hohen Wert (Mittelwert plus eine Standardabweichung) zeigt sich hingegen ein positiver Zusammenhang. Mit dieser Darstellungsform lässt sich der konditionale Zusammenhang von $x_{i}$ und $y_{i}$ über den Wertebereich von $z_{i}$ gut veranschaulichen.

Es sei erwähnt, dass die Berechnung marginaler Effekte oder vorhergesagter Werte auch möglich ist, wenn die Wertebereiche der beteiligten Variablen die 0 nicht 


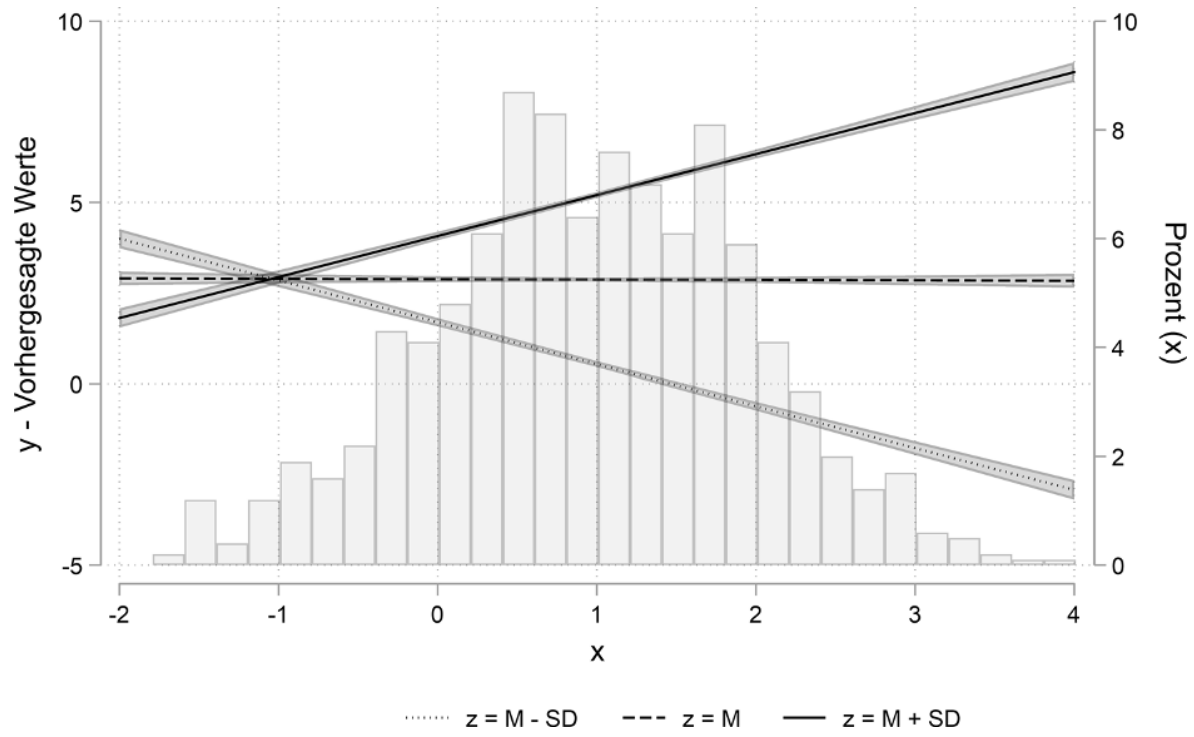

Abb. 2 Vorhergesagte Werte für y aus Modell 2 (Tab. 1) und Verteilung von x

umfassen und die Variablen nicht zentriert wurden, solange ein inhaltlich sinnvoller Wertebereich gewählt wird. Die Wahl eines sinnvollen Wertebereichs ist auch für die Bewertung der statistischen Bedeutsamkeit von Relevanz, da die Standardfehler und entsprechend die statistischen Irrtumswahrscheinlichkeiten der Effekte ebenfalls über den Wertebereich der Variablen variieren können (wie an der unterschiedlichen Breite der Konfidenzintervalle in Abb. 2 ersichtlich). Es macht daher auch wenig Sinn, lediglich anhand der dargestellten Ergebnisse in einer Regressionstabelle zu beurteilen, ob der Haupteffekt von $x_{i}$ einen statistisch signifikanten Effekt auf $y_{\mathrm{i}}$ ausübt, solange ein von 0 abweichender Interaktionseffekt vorhanden ist (Allison 1977; Brambor et al. 2006, S. 73 f.). ${ }^{5}$

Anhand von Abb. 2 lässt sich auch auf anderem Wege nachvollziehen, dass eine unabhängige Interpretation von Haupt- und Interaktionseffekten wenig zielführend ist; egal ob die zugrunde liegenden Daten zentriert oder unzentriert sind. Durch die Zentrierung verändert sich nämlich die substanzielle Bedeutung des Modells nicht (was auch Mayerl und Urban 2019 hervorheben, siehe bspw. S. 146), sondern lediglich die Interpretation der Haupteffekte, weil $\beta_{2}$ und $\beta_{3}$ nun andere Effekte in den Daten modellieren. Der Interaktionseffekt (einschließlich des Standardfehlers) bleibt gegenüber dem unzentrierten Modell, ebenso wie die vorhergesagten Werte, unverändert. Das zentrierte und unzentrierte Modell sind statistisch äquivalent (aus-

\footnotetext{
5 Aussagen über die statistische Signifikanz sind natürlich besonders uninformativ, wenn in der Realität der Wert 0 bei $x_{i}$ gar nicht vorkommt. Aber auch im Fall der Mittelwertzentrierung ist zu beachten, dass sich die statistische Signifikanz lediglich auf den Mittelwert in den Daten oder auf den durchschnittlichen Effekt von $x_{i}$ über den gesamten Wertebereich von $z_{i}$ bezieht.
} 
führlicher in Aiken und West 1991; Dalal und Zickar 2012). ${ }^{6}$ Entsprechend ließe sich auch mit dem zentrierten Modell Abb. 2 erstellen, wenn die korrespondierenden, d.h. zentrierten, Werte für $z_{i}$ verwendet werden. Somit bleibt, unabhängig von der Zentrierung, die Implikation der konditionalen Interpretation erhalten: Hauptund Interaktionseffekte können nicht unabhängig voneinander sinnvoll interpretiert werden. Aus diesem Grund raten wir von der Mittelwertzentrierung ab, wenn eine (grafische) Darstellung mithilfe vorhergesagter Werte oder marginaler Effekte angestrebt wird: Es ist ein unnötiger Zwischenschritt, der einen weiteren Zwischenschritt notwendig macht (die Umrechnung der ausgewählten Werte von $z_{i}$ auf der ursprünglichen Skala in die mittelwertzentrierte Skala).

Die Berechnung vorhergesagter Werte oder marginaler Effekte auf Basis eines inhaltlich sinnvollen Wertebereichs schließt zudem an die methodische Diskussion nichtlinearer Modelle (bspw. Logit-Modelle) mit Interaktionseffekten an (z. B. Ai und Norton 2003; Bauer 2010; Long und Freese 2014). Hier ist im Unterschied zu linearen Modellen bereits im additiven Modell (ohne multiplikativen Interaktionsterm) aufgrund der Nichtlinearität des Modells eine Interpretation der Effekte als unkonditionale oder durchschnittliche Marginaleffekte nicht möglich (Long und Freese 2014; Brambor et al. 2006, S. 72). Entsprechend komplexer stellt sich dann die Interpretation der Effekte in nichtlinearen Regressionen dar, wenn konditionale Hypothesen im Rahmen von Interaktionsmodellen getestet werden, weshalb in der entsprechenden Literatur eine Berechnung der vorhergesagten Wahrscheinlichkeiten oder der Marginaleffekte auf die Wahrscheinlichkeiten und ihre grafische Darstellung als unumgänglich erachtet wird (Bauer 2010; Brambor et al. 2006; Long und Freese 2014; Nisic 2018). ${ }^{7}$

King et al. (2000) haben bereits vor fast 20 Jahren dafür plädiert, die Ergebnisse quantitativer Analysen so zu präsentieren, dass sie verständlich und transparent sind: ,Yet statistical analysts have a responsibility to present their results in ways that are transparent to everyone. In too much research, understanding even the substantive conclusions of sophisticated quantitative models can be challenging at best and impossible at worst."

Für den praktischen Umgang mit Interaktionen, sowohl in linearen als auch in nichtlinearen Modellen, lassen sich daher folgende Hinweise bezüglich der Interpretation und Darstellung geben (Allison 1977; Brambor et al. 2006; Berry et al. 2012; Clark et al. 2006):

1. Haupteffekte (und Interaktionseffekte) können nicht wie unkonditionale marginale Effekte interpretiert werden.

2. Haupt- und Interaktionseffekte sollten nicht isoliert voneinander interpretiert werden.

3. Haupt- und Interaktionseffekte sollten gemeinsam dargestellt und interpretiert werden, bspw. durch vorhergesagte Werte oder marginale Effekte.

\footnotetext{
${ }^{6}$ Dies gilt insbesondere für das Standard OLS-Modell. Allerdings gibt es durchaus Anwendungsfälle, wie z. B. Mehrebenmodelle, in denen die Zentrierung von Variablen durchaus von methodischer Bedeutung ist. Siehe hierzu die entsprechende Fachliteratur (Enders und Tofighi 2007; Schunck 2013; Schunck und Perales 2017).

7 Dabei ist bei nicht-linearen Modellen zu beachten, dass sich AME und MEM - anders als in den hier besprochenen linearen Modellen - nicht zwingend entsprechen müssen.
} 
Danksagung Für wertvolle Hinweise und Kommentare danken wir Sebastian Bähr, Daniel Baron, Fabian Ochsenfeld, Klaus Pforr und Carsten Sauer. Für alle verbleibenden Mängel sind die Autor*Innen verantwortlich

Funding Open Access funding provided by Projekt DEAL.

Open Access Dieser Artikel wird unter der Creative Commons Namensnennung 4.0 International Lizenz veröffentlicht, welche die Nutzung, Vervielfältigung, Bearbeitung, Verbreitung und Wiedergabe in jeglichem Medium und Format erlaubt, sofern Sie den/die ursprünglichen Autor(en) und die Quelle ordnungsgemäß nennen, einen Link zur Creative Commons Lizenz beifügen und angeben, ob Änderungen vorgenommen wurden.

Die in diesem Artikel enthaltenen Bilder und sonstiges Drittmaterial unterliegen ebenfalls der genannten Creative Commons Lizenz, sofern sich aus der Abbildungslegende nichts anderes ergibt. Sofern das betreffende Material nicht unter der genannten Creative Commons Lizenz steht und die betreffende Handlung nicht nach gesetzlichen Vorschriften erlaubt ist, ist für die oben aufgeführten Weiterverwendungen des Materials die Einwilligung des jeweiligen Rechteinhabers einzuholen.

Weitere Details zur Lizenz entnehmen Sie bitte der Lizenzinformation auf http://creativecommons.org/ licenses/by/4.0/deed.de.

\section{Literatur}

Ai, Chunrong, und Edward C Norton. 2003. Interaction terms in logit and probit models. Economics letters 80:123-129.

Aiken, Leona S., und Stephen G. West. 1991. Multiple regression: testing and interpreting interactions. Newbury Park: Sage Publications.

Allison, Paul D. 1977. Testing for interaction in multiple regression. American Journal of Sociology 83:144-153.

Balli, Hatice Ozer, und Bent E Sørensen. 2013. Interaction effects in econometrics. Empirical Economics 45:583-603.

Bauer, Gerrit. 2010. Graphische Darstellung regressionsanalytischer Ergebnisse. In Handbuch der sozialwissenschaftlichen Datenanalyse, Hrsg. Christof Wolf und Henning Best, 905-927. Wiesbaden: VS Verlag für Sozialwissenschaften.

Berry, William D., Matt Golder und Daniel Milton. 2012. Improving tests of theories positing interaction. The Journal of Politics 74:653-671.

Bischof, Daniel. 2017. New graphic schemes for Stata: plotplain and plottig. The Stata Journal 17:748-759.

Brambor, Thomas, William Roberts Clark und Matt Golder. 2006. Understanding Interaction Models: Improving Empirical Analyses. Political Analysis 14:63-82.

Braumoeller, Bear F. 2004. Hypothesis testing and multiplicative interaction terms. International Organization.

Cameron, A Colin, und Pravin K Trivedi. 2005. Microeconometrics: methods and applications. Cambridge: Cambridge University Press.

Clark, William Roberts, Michael J Gilligan und Matt Golder. 2006. A simple multivariate test for asymmetric hypotheses. Political Analysis 14:311-331.

Cohen, Jacob, Patricia Cohen, Stephen G West und Leona S Aiken. 2003. Applied multiple correlation/ regression analysis for the social sciences. 3. Aufl. Mahwah: Lawrence Erlbaum Associates.

Dalal, Dev K., und Michael J. Zickar. 2012. Some common myths about centering predictor variables in moderated multiple regression and polynomial regression. Organizational Research Methods 15:339-362.

Enders, Craig K., und Davood Tofighi. 2007. Centering predictor variables in cross-sectional multilevel models: a new look at an old issue. Psychological Methods 12:121.

King, Gary, Michael Tomz und Jason Wittenberg. 2000. Making the most of statistical analyses: Improving interpretation and presentation. American Journal of Political Science 44:347-361.

Long, J. Scott, und Jeremy Freese. 2014. Regression models for categorical dependent variables using Stata. College Station, TX: Stata Press.

Mayerl, Jochen, und Dieter Urban. 2019. Vorsicht (!) bei Regressionsanalysen mit Interaktionsvariablen. KZfSS Kölner Zeitschrift für Soziologie und Sozialpsychologie 71:135-156. 
Mitchell, Michael N. 2008. A visual guide to Stata graphics. College Station, TX: Stata Press.

Nisic, Natascha. 2010. Mitgegangen - Mitgefangen? Die Folgen von Haushaltsumzügen für die Einkommenssituation von Frauen in Partnerschaften. Kölner Zeitschrift fur Soziologie und Sozialpsychologie 62:515-549.

Nisic, Natascha. 2018. Die Auslagerung familiärer Sorgearbeit als Vertrauensproblem - Eine wirtschaftssoziologische Betrachtung der Nachfrage nach Haushaltshilfen und Kinderbetreuung. Zeitschrift für Soziologie 47:219-239.

Schunck, Reinhard. 2013. Within and between estimates in random-effects models: Advantages and drawbacks of correlated random effects and hybrid models. Stata Journal 13:65-76.

Schunck, Reinhard. 2016. Reich und schön? Eine Untersuchung zur ungleichen Verteilung physischer Attraktivität. Kölner Zeitschrift für Soziologie und Sozialpsychologie 68:113-137.

Schunck, Reinhard, und Francisco Perales. 2017. Within-and between-cluster effects in generalized linear mixed models: A discussion of approaches and the xthybrid command. Stata Journal 17:89-115.

Reinhard Schunck Dr., Professor für Soziologie. Forschungsschwerpunkte: Soziale Ungleichheit, Migration, Familie, Gesundheit, Lebenslauf, quantitative Methoden. Veröffentlichungen, u. a.: Motherhood and mental well-being in Germany: Linking a longitudinal life-course design and the gender perspective (mit M. Giesselmann, M. Hagen). Advances in Life Course Research 37, 2018; A Relational Inequality Approach to First-and Second-Generation Immigrant Earnings in German Workplaces (mit S. M. Melzer, D. Tomaskovic-Devey, P. Jacobebbinghaus). Social Forces 97, 2018.

Natascha Nisic Dr., Univ.-Professorin für Lebensführung und Sozioökonomie des privaten Haushalts. Forschungsgebiete: Wirtschafts-, Arbeitsmarkt- und Familiensoziologie, Methoden der empirischen Sozialforschung, Soziale Ungleichheit. Aktuelle Publikationen: Die Auslagerung familiärer Sorgearbeit als Vertrauensproblem - Eine wirtschaftssoziologische Betrachtung der Nachfrage nach Haushaltshilfen und Kinderbetreuung. Zeitschrift für Soziologie 53, 2018; Smaller Differences in Bigger Cities? -Assessing the Regional Dimension of the Gender Wage Gap. European Sociological Review 33, 2017; Explaining Gender Inequality That Follows Couple Migration (mit S. Melzer), Journal of Marriage and Family 78, 2016. 